\title{
Empatía como emoción emergente en el proceso de enseñanza y aprendizaje
}

\author{
Empathy as an emerging emotion in the teaching and learning process
}

A empatia como emoção emergente no processo de ensino e aprendizagem

\author{
Daniela Barría Díaz \\ Magister en educación, mención gestión y política \\ Docente Instituto de Ciencias de la Educación \\ Facultad de Filosofía y Humanidades \\ Universidad Austral de Chile \\ dbarriadiaz@gmail.com
}

\section{Resumen}

Los procesos educativos conllevan a diversas emociones que emergen desde la relacionalidad. En muchas ocasiones, estás emociones se ven negadas debido a las exigencias propias del sistema educativo. Escasos espacios de reflexión y de retroalimentación provocan un sin sentido del quehacer educativo. La posibilidad de dar lugar a la emergencia de nuestras emociones, permite la validación del estar con otro. Una de las emociones que permite y responde tanto en el quehacer de la vida cotidiana como también en los procesos educativos es la empatía. Reflexionar acerca de la emoción de la empatía, sus dimensiones desde lo biológico, el lenguaje y la educación nos permiten un mayor acercamiento a la relación con el otro, a poder comprenderse a través de la conciliación de nuestras posturas, de permitirnos ser en el otro, y por sobre todo crecer de manera colectiva, dialógica y con apertura de nuestras emociones.

Palabras claves: Empatía, emociones y Relacionalidad.

\section{Summary}

The educational processes lead to diverse emotions that emerge from the relationality. On many occasions, these emotions are denied due to the demands of the educational system. Scarce spaces for reflection and feedback cause a senseless educational work. The possibility of giving rise to the emergence of our emotions, allows the validation of being with another. One of the emotions that allows and responds both in the task of everyday life and in educational processes is empathy. Reflect on the emotion of empathy, its dimensions from the biological, language and education allow us a closer approach to the relationship with the other, to be understood through the reconciliation of our positions, allowing us to 
be in the other, and above all grow collectively, dialogically and with openness of our emotions.

Keywords: Empathy, emotions and relationality.

\section{Resumo}

Os processos educacionais levam a diversas emoções que emergem da relacionalidade. Em muitas ocasiões, essas emoções são negadas devido às demandas do sistema educacional. Espaços escassos para reflexão e feedback causam um trabalho educacional sem sentido. A possibilidade de dar origem ao surgimento de nossas emoções, permite a validação de estar com outra. Uma das emoções que permite e responde tanto na tarefa do cotidiano quanto nos processos educacionais é a empatia. Reflita sobre a emoção da empatia, suas dimensões da biologia, da linguagem e da educação nos permitem uma aproximação mais próxima do relacionamento com o outro, para ser entendida através da conciliação de nossas posições, permitindo-nos estar no outro, e sobretudo crescem coletivamente, dialógicamente e com a abertura de nossas emoções.

Palavras chaves: Empatia, emoções e relacionalidade.

\section{La empatía como fenómeno biológico}

La empatía como definición etimológica significa "cualidad de sentirse dentro". Luego a través de la historia se han acuñado diversas definiciones entre ellas una que integró una perspectiva cognitiva y afectiva fue la propuesta por Salovey en Fernández y otros, (2008) cuando afirman que "la empatía requiere la adecuada identificación de las respuestas emocionales en otras personas e implica no sólo actitudes sino también habilidades o competencias bien definidas".

Comprender la empatía como fenómeno biológico, aborda dimensiones que van más allá de la individualidad del comportamiento humano, es así como menciona Toro (2005, p. 131), que "es una manifestación de la integralidad sistémica del mismo" y así como también involucra la sutileza del fenómeno desde una perspectiva ecológica, donde el centro de esto, es la interacción entre los individuos y su ecosistema, es decir, la relación entre ellos y el medio que nos circunda. Igualmente como De Waal señala en distintas publicaciones menciona una serie de ejemplos que fueron observados por él donde los animales presentaban conductas que no sólo era una socialización entre ellos, sino que comprende conductas que logran empatizar entre ellos y que por ende los conduce a una mejor adaptación ${ }^{1}$ y/o evolución ${ }^{2}$. Frente a este argumento De Waal (2006, p. 53), menciona que "la empatía es precisamente uno de esos mecanismos, donde la selección debe haber favorecido aquellos mecanismos que evalúen los estados emocionales de los otros y respondan con rapidez a los mismos, ya que así como se hace mención a las emociones, cabe señalar

\footnotetext{
1 Adaptación: estado de encontrarse ajustado al ambiente como resultado de la selección natural. Curtis et.al. 2000, p. G1).

2 Evolución: cambios en el reservorio genético de una generación a la siguiente como consecuencias de procesos como la mutación, la selección natural, al apareamiento no aleatorio y la deriva génica. (Curtis et. al. 2000, p. G11)
} 
que desde el origen del hombre somos considerados por esencia que somos animales grupales: altamente cooperativos, sensibles a la injusticia, a veces beligerantes, pero principalmente amantes de la paz" (De Waal, 2011, p. 19). En este sentido la naturaleza nos muestra distintos comportamientos donde el desarrollo de competencias sociales y el vínculo entre ellos comprenden más que una colaboración, así mismo De Waal (2011, p. 27) manifiesta que "la vinculación tiene para nosotros un inmenso valor de supervivencia, y el vínculo más crítico es el que se establece entre madre e hijo. Este vínculo proporciona la plantilla evolutiva para los otros vínculos, incluso entre adultos, por ende la vinculación es esencial para nuestra especie, y es lo que nos hace más felices". Bajo esta óptica la supervivencia ligada a la relación o vínculo que se genera entre madre e hijo, es uno de los primeros eslabones de la socialización en que se incluyen aspectos emocionales entre uno y el otro. Considerar la emergencia de las emociones al estar en contacto o sintonía con el otro, comprende un lineamento en la construcción del concepto de empatía. Es así como De Waal (2006: 49) comenta que "existiendo una creciente diferenciación entre el yo y el otro, así como una creciente apreciación de las circunstancias precisas que subyacen en los estados emocionales de los demás, el contagio emocional se convierte en empatía. La empatía comprende y no podría haber surgido sin el contagio emocional". Desde esta mirada el autor citado hace una referencia de cómo se genera la empatía, si bien hace mención al contagio emocional que valida las situaciones vivenciadas en relación de uno al otro.
Igualmente Petrillo (2009, p. 369) menciona que "la empatía consiste poner al yo extraño en el modo de un copresente", es decir, un tiempo común en que los individuos (yo- el otro) comparten un espacio donde emergen sus emociones en el momento vivenciado. De esta manera Quintanilla (2004, p. 91) señala que la idea de "la empatía es el intentar alejarnos de nuestros propios estados mentales para imaginar ser el agente, dejando espacio en nosotros mismos para albergar al otro en su particularidad", realizando un acto de amor en dejar que el otro surja con su propia esencia, desde aquí cabe comprender la situación ${ }^{3}$ o más bien como nos situamos con el otro, es decir, según Casassus (2007, p. 190), "la realidad que vive una persona y en la cual ella se encuentra, es una situación que está en relación a esa persona y a lo que esa persona haga". Así mismo la empatía conlleva a una proyección en la situación del otro, haciendo relación al tiempoespacio, que como menciona Petrillo, (2009, p. 369), "el tratamiento de la estructura de la empatía está estrechamente ligado con la constitución tanto de la temporalidad propia como de la vida extraña”.

\section{Dimensiones de la empatía}

Por otro lado, otra dimensión para comprender es la comunicación ${ }^{4}$ empática que aborda aspectos como la "comprensión emocional que consiste en estar abierto a la otra persona y tener la capacidad de ponerse en su lugar" (Casassus, 2007, p. 187). Para lograr esta

\footnotetext{
3 Según Casassus (2007, p. 190), situación, es estar en un "lugar" donde "algo" está situado.

4 La comunicación con otro, es un intercambio de información con sentido con ese otro. Este intercambio puede ser de muchas maneras: puede ser lingüístico, o gestual, o corporal. (Casassus, 2007, p. 188)
} 
apertura y poder empatizar, hay que considerar, la cultura y el lenguaje que dan forma a las expresiones empáticas. La distinción entre "ser el origen de y dar forma a, es esencial, y aquí sostendré que la empatía es la forma original y prelinguística de vinculación interindividual que sólo de forma secundaria se ha visto sometida a la influencia del lenguaje y cultura". (De Waal, 2006, p. 49). Indudablemente "el lenguaje, en el más amplio sentido de la palabra se convierte en nuestra capacidad distintiva como especie y al mismo tiempo como la generadora de nuestro desarrollo" (Toro, 2005, p. 128).

Por otro parte, las actitudes o conductas que se generan a partir de la relación empática entre las personas, pueden ser caracterizadas por distintos autores, es así como Moreno y Fernández (2010, p. 42) señalan que "la capacidad de ponerse en el lugar del otro y la capacidad de ajustar los propios pensamientos, sentimientos y conductas a situaciones y condiciones cambiantes, posibilitan el establecimiento de actitudes prosociales, entre las cuales la capacidad de perdonar es una de las más importantes para lograr la armonía social. Bajo esta óptica la empatía motiva a uno a cambiar desde el foco propio de la experiencia dolorosa hacia el foco centrado en el otro, supone el cuidado y el tener en cuenta las necesidades del ofensor y, en este sentido, la consideran la variable mediadora más importante que explica la capacidad de perdonar". (Moreno y Fernández, 2010, p. 44). Del mismo modo, comprender la dimensionalidad de la empatía sujeta a las conductas que se pueden generar es lo que conlleva como bien menciona Quintanilla (2004, p. 82), quien señala "que entender al otro no sería reconstruir su vida mental, ni conocer los estados mentales que causaron sus acciones, sino generar una forma de vida común, una intersección de horizontes que conlleva a una invitación al cambio, la tolerancia y la adaptación, ya que a mi modo de ver, todo pasa por cada uno de nosotros, es así como el cuidado por uno mismo, como la auto-comprensión pasa por el cuidado y comprensión del otro". (Quintanilla, 2004, p. 94).

Así mismo como bien se señala anteriormente algunas conductas generadas a través de la empatía, también se puede definir como "el principio de caridad $^{5}$, donde uno permite la comprensión del otro, pero también hace posible la comprensión de sí mismo, su auto-descripción y, por tanto, también su transformación". (Quintanilla, 2004, p. 95), igualmente desde este "principio de caridad se deduce una obligación moral de tratar de entender al otro, de intentar incorporarlo a las posibilidades de nuestro propio discurso" (Quintanilla, 2004, p. 94). Sujeto a esta cita, más que una obligación es dar un sentido a la intención de la relacionalidad entre los sujetos, considerar aspectos como "del mismo modo que mi pasado presupone mi presente viviente, presupone la presentación del co-presente extraño también un presente viviente extraño" (Petrillo, 2009, p. 372).

\section{La Empatía como lenguaje}

Cada definición de empatía por parte de los distintos autores, si bien, coinciden en algunos aspectos, cada uno establece distintas dimensiones o aspectos que son fortalecidos en su lenguaje, es así como Toro, (2005, p. 130) clasifica distintos

\footnotetext{
5 La caridad es el cuidado desinteresado por el otro, pero es también el cuidado de uno mismo, y ambas formas de cuidado son inseparables. (Quitanilla, 2004, p. 94)
} 
casos de empatía, uno de ellos es la empatía "fundada en la percepción que se construye a partir de la presencia corpórea del otro y reconociendo la integralidad del otro, vale decir desde un principio de intención comprender al otro desde la situación, asimismo el segundo tipo de empatía, la define como una imaginaria transposición de sí mismo al lugar del otro, es más activa y compromete más aspectos de carácter cognitivo que el tipo anterior, el tercer tipo de empatía, relacionada con la comprensión del tu como otro hacia mí y del mí como otro hacia el tú, se orienta hacia la idea básica de entender mi propia situación desde la perspectiva del otro que está en relación y viceversa". (Toro, 2005, 131), y por "último este tipo de empatía se traduce en la capacidad de comprender que el otro es tan digno como yo para estar en lugar o espacio que habita y su presencia no es prescindible" (Toro, 2005, p. 132).

Bajo estas concepciones se contempla que las "dimensiones de la empatía son: sentir lo que el otro siente, conocer lo que el otro está sintiendo y responder con comprensión a la situación de la otra persona". (Toro, 2005, p. 130). Es así como Garaigordobil \& Maganto, (2011, p. 257) señalan que la "empatía es un factor importante en el proceso en que el individuo desarrolla pautas de pensamiento y comportamiento acordes con las normas sociales". Si bien se puede comprender de conductas propias a las normas sociales, la empatía transciende, ya que en tal sentido esta requiere en primer lugar de una plena implicación emocional.

Considerando de esta manera la relevancia que conlleva el estar conscientes emocionalmente, así mismo, "contemplar las emociones ajenas despierta nuestras propias emociones, y de aquí construimos una comprensión más profunda de la situación del prójimo" (De Waal, 2011, p. 103), y bajo esta misma óptica Quitanilla (2004: 95) indica que la empatía es "interpretar al otro no es reconstruir sus contenidos mentales en los nuestros, como sostendría una concepción internalista de la mente, sino más bien es la actividad creativa de construir un territorio compartido: una comunidad de creencias, deseos, significados, valores y objetos de la realidad". Del mismo modo Toro (2005, p. 130) complementa que "la empatía se genera en todo el actuar de la persona, en todo lo que desprende y es observable como en todo aquello que internaliza y no es observable o por lo menos conscientizable desde el punto de la vista de la expresión verbal o del análisis explícito. De tal manera que al sentir lo que el otro siente, conocer lo que el otro está sintiendo y responder con comprensión a la situación de la otra persona, la empatía adquiere un estatus de percepción-acción".

Por otra parte, cabe mencionar ¿de qué manera se acciona la empatía entre los individuos?, ¿qué estímulo provoca en el ser propio una transposición del otro?, y es a partir de esto que no se puede dejar de mencionar la expresión del cuerpo ya que como menciona Varela (2000, p. 106), "lo mental es algo que surge de los afectos y las emociones, y estos, a su vez anclados en el cuerpo". Así mismo nuestro cuerpo manifiesta nuestras emociones, de tal manera que como señala De Waal, (2011, p. 87) "el cuerpo genera sensaciones internas y se comunica con otros cuerpos, lo que nos sirve para construir relaciones sociales y apreciar la realidad circundante. Puesto a que ya no es posible pensar en la mente sin que ella esté totalmente encarnada con 
el cuerpo físico. Nuestra mente sigue las fluctuaciones del cuerpo". (Casassus, 2007, p. 75). En este mismo sentido Toro, (2005, p. 130) señala que "la empatía en algunos casos, está fundada en la percepción que se construye a partir de la presencia corpórea del otro y reconociendo la integralidad del otro, vale decir desde un principio de intención de comprender la otro desde su situación, por lo tanto es más que inferir asépticamente su comportamiento desde su comportamiento observable, sino más bien es intencionar un acto de situarse desde la perspectiva del otro". Igualmente Casassus (2007, p. 75) comenta que "el cuerpo es una mente densa, que percibe para que la mente pueda realizar su acto de percepción más fina y actuar. El cuerpo es un objeto de percepción de la mente. El cuerpo es un órgano de expresión y percepción". Si bien el cuerpo como entidad completa refleja la resonancia de nuestras emociones, cabe señalar que nuestro rostro o cara es el puente hacia las percepciones de los otros, es así como De Waal, (2011, p. 116) señala que "la cara sigue siendo la autopista de la emoción, la conexión más rápida con los otros".

De esta misma forma De Waal, postula una teoría para comprender de qué manera las emociones ajenas pueden afectar las propias y para ello fundamenta la importancia que radica la comunicación corpórea, donde menciona la Teoría de la prioridad del cuerpo, sostiene que "el punto de partida del cuerpo y que las emociones vienen después. El lenguaje corporal de alguien afecta a nuestro propio cuerpo, cuyo eco emocional nos hace sentir en consecuencia” (Waal, 2011, p. 114). Así mismo desde el propio lenguaje corporal como también el contacto corporal es fundamental en las relaciones, ya que el "contacto corporal para confortar a otros forma parte de nuestra biología mamífera y se remota a la lactancia y los cuidados maternales" (Waal, 2011, p. 128). Es así como frente a una situación de aflicción, de manera que uno empatiza, surge de manera innata la conducta de que "si sientes que otro sufre, ve y entabla contacto con él" (Waal, 2011, p. 130).

\section{Empatía y educación}

Finalmente abordar la empatía como plataforma sustancial en el ámbito de la Educación, es generar en sí dimensiones fundamentales en relación entre profesor(a) y alumno(a). Por esta razón comprender la empatía en el ámbito de la educación invita a dimensionar la tarea de educar como un espacio de convivencia; así mismo Maturana, (1999, p. 151) señala que como rol fundamental el "educador o la educadora es aquel o aquella que adopta la tarea de configurar un espacio de convivencia donde otros se transforman con él o ella. Para que esto pase, el educando $\mathrm{y}$ el educador $\mathrm{o}$ educadora, deben acceder al espacio en que se aceptan mutuamente como legítimos otros en la convivencia”. De igual manera Toro, (2005, p. 35) menciona que "un profesor empático no es un discurso, para comenzar no podría ser profesor (el que profesa) sino más bien el que favorece, crea o desarrolla ambientes de aprendizaje intencionados, que co-construye, construyendo y al mismo tiempo se construye. El que media o favorece el intercambio de los sentidos entre los sujetos y la cultura". Y bajo esta misma mirada Maturana, (1999, p. 59), afirma que "la tarea de la educación es crear un espacio relacional en el que nuestros niños crecen ahora, en el presente, como seres humanos responsables ecológica y socialmente que se respetan a sí mismos". 
Comprender y estar consciente de fomentar la capacidad empática entre los educandos contribuye de alguna manera "porque aporta información sobre la resolución de conflictos en edades tempranas" (Garaigordobil \& Maganto, 2011, p. 264), como también "la empatía tenga un mayor efecto inhibidor frente a las respuestas más agresivas" (Moreno y Fernández, 2010, p. 50). Así mismo la empatía contribuye según estudios de Garaigordobil \& Maganto, (2011, p. 264) a "que adolescentes con alta empatía también usaban muchas estrategias cooperativas de resolución de conflictos y pocas agresivas. Incluso en la adolescencia temprana la conexión entre la capacidad de resolver constructivamente conflictos y la empatía, factor determinante en el proceso en el que el individuo desarrolla pautas de pensamiento y comportamiento relacionados con las relaciones interpersonales". De modo similar, "a mayor capacidad empática por parte de los alumnos, se observó una menor posibilidad de dar una respuesta agresiva ante un ofensor (en particular de la respuesta de venganza) y que es mayor la probabilidad de una respuesta prosocial (en particular del perdonar)" (Moreno y Fernández, 2010: 41). Junto con ello comprender la empatía en toda su dimensión, puede "facilitar el diseño de programas y estrategias educativas que incidan directamente sobre el desarrollo de dicha variable relacionada con la resolución positiva de conflictos y alternativas menos agresivas de respuesta ante la frustración y el conflicto" (Garaigordobil \& Maganto, 2011, p. 265).

La empatía a través de sus distintas dimensiones, manifiesta en primer lugar que somos individuos que siempre estamos sociabilizando y que en muchos casos actuamos en sincronía ${ }^{6}$, en este sentido De Waal (2011, p. 41), señala que "somos animales grupales hasta la saciedad, por ende siempre ha existido una preocupación por el que está a nuestro lado, por tanto, la capacidad de funcionar en grupo y construir una red de ayuda mutua es crucial para la supervivencia" (De Waal, 2011, p. 54). Vale decir, que desde las manifestaciones de nuestros antepasados como sujetos grupales-sociales, se destaca la dimensión de individuos cooperativos y sincronizados en su acción. En esta perspectiva hablando desde una mirada evolutiva, en tiempos de hoy se ha perdido este fenómeno ya que ha surgido el individualismo y la indiferencia ${ }^{7}$, siendo patrones que han movilizado a nuestra sociedad, y es así como estos aspectos provocan una involución con respecto a nuestras relaciones como individuos. Por lo mismo Maturana, (1999, p. 25), señala que aspectos como la indiferencia ocurre cuando, "el otro no tiene presencia, y lo que le suceda a él o ella está fuera del dominio de nuestras preocupaciones", no es más que emociones que surgen en la vida diaria y que no condicen con la esencia de nuestros antepasados que nos mueve como sociedad. Así mismo De Waal (2011, p. 53), señala que la "lucha por la existencia no es tanto una lucha de todos contra todos, sino más bien de masas de organismos contra un entorno hostil". Vale decir, existe una lógica de comportamiento, que tiene una objetividad en cuanto se tengan necesidades en una sociedad, por lo tanto abordar dimensiones de la empatía como

\footnotetext{
6 La sincronía, a su vez, se asienta en la capacidad de ponerse en la piel del otro y hacer propios los movimientos ajenos. (De Waal, 2011, p. 78).

7 La indiferencia es el domino de aquellas conductas relacionales a través de las cuales el otro no es visto como otro. (Maturana, 1999, p. 45).
} 
acto fundamental en nuestras acciones, lo posiciona desde una perspectiva "holística donde consideraría inevitable el desear comprender al otro y a uno mismo, mediante el reconocimiento de nuestro espacio compartido, como un solo proceso que es condición de posibilidad de toda acción en el mundo". (Quitanilla, 2004, p. 94).

\section{Conclusión}

La empatía desde sus múltiples dimensiones, despliega emociones que posibilitan el proceso pedagógico, por ejemplo a través del desarrollo humano, el manejo de conflictos, la conciencia de lo aprendido, entre otros. Es por ello que desde la mirada de la relacionalidad entre profesor-alumno, es decir, en el ámbito de lo pedagógico, nos favorece en torno a la construcción del conocimiento, puesto a que de esta manera se brinda un espacio de co-emergencia del yo y de los otros y en el mismo sujeto.

Por consiguiente, educar en base a la empatía, establece una plataforma de dinámicas relacionales que buscan comprender al otro como así mismo, una emergencia de esta emoción, que se lleva no solo a ponerse en lugar del otro, sino más bien a generar espacios dialógicos, comprensivos, de apertura, de reflexión, dimensiones que son fundamentales en el proceso de enseñanza-aprendizaje. Es así como Varela, (2010:252), señala "que un aspecto esencial de la empatía, el camino real para acceder a la vida social consciente, más allá de la simple interacción". Esto nos otorga la oportunidad de estar consciente de nuestra relación con los demás, y además concede herramientas para dar sentido a nuestras experiencias de vidas. Por ello, la invitación al ser empático, comprende no solo a ponerse en el lugar del otro, "sino más bien es la actividad creativa de construir un territorio compartido: una comunidad de creencias, deseos, significados, valores y objetos de la realidad" (Quintanilla 2004, p. 95).

Además, cabe mencionar que como animales gregarios, que responden a fenómenos colectivos, no pueden haberse dado sin un proceso interno que se refleja en el otro como el ser empático. Vale decir, como sujetos en crecimiento interno de auto formación, que sin este proceso se carece de un sentido de reflejo y de proyección en el otro.

En tal sentido, no podemos dejar de mencionar un proceso fundamental que se denota primeramente en el yo presente, que es la autopoéisis, como la "autonomía de lo vivo, la organización de lo vivido como configuración, organización de lo vivido, mecanismo de constitución de su identidad" (Maturana y Varela, 2008, p. 45). De esta manera se analiza este proceso como la capacidad de un individuo en autoformarse, vale decir a partir de su propio ser, se autoconstruye, se configura a través del medio y de su influencia y por otro lado se destaca que a pesar de ser individuo en relación, tiene propiedades únicas que lo definen como un ser autónomo. Además asociado como ser biológico que evoluciona, que se genera del ser sin perder su identidad y autonomía.

\section{Referencias}

Abarca, M., Marzo, L. y Sala, J. (2002). La educación emocional y la interacción Profesor/a-Alumno/a. Revista Electrónica Interuniversitaria de Formación del Profesorado. 5(3).

Abarca, M., Marzo, L. y Sala, J. (2002). La educación emocional y la 
interacción Profesor/a-Alumno/a. Revista Electrónica Interuniversitaria de Formación del Profesorado. 5(3).

Ascorra, P., Arias, H. y Graff, C. (2003). La escuela como contexto de contención social y afectiva. Departamento de Educación, Facultad de Ciencias Sociales, Universidad de Chile. Rev. Enfoques Educacionales, vol.5, No.1, 117-135.

Buxarris, M. \& Martínez, M. (2009). Educación en valores y educación emocional: propuestas para la acción pedagógica. Revista electrónica Teoría de la Educación. Educación y Cultura en la Sociedad de la información. Vol.10. No.2, 265-275.

Briones, G. (2002). Epistemología de las Ciencias Sociales. ARFO Editores e Impresores Ltda. Bogotá.

Canales, M. (2006). Metodologías de investigación social. Inducción a los oficios. LOM Ediciones. Santiago.

Casassus, J. (2007). La educación del ser emocional. Editorial Cuarto Propio. Santiago de Chile.

Castillo, I. y otros. (2010). Pedagogía, diversidad y lenguaje: develando los colores en miradas aprendientes. Universidad Nacional, Costa Rica, Rev. Electrónica Educare, vol. XIV, núm. 1, enero-junio, 2010, pp. 85-95.

De Waal, F. (2011). La edad de la empatía, ¿somos altruistas por naturaleza? Tusquets Editores, S.A. Barcelona.

Denzin, N. y Lincoln, Y. (2012). Manual de la investigación cualitativa. Editorial Gedisa. Barcelona.
Fernández y otros. (2008). Empatía: Medidas, teorías y aplicaciones en revisión. Anales de psicología. Servicio de Publicaciones de la Universidad de Murcia. Murcia (España). Vol. 24, No. 2, 284-298.

Fontaine, I. (2000). Experiencia emocional, factor determinante en el desarrollo cerebral del niño/a pequeño/a. Universidad Austral de Chile. Facultad de Filosofía y Humanidades, Rev. Estudios Pedagógicos. No. 26, 119-126. Valdivia.

Guber, R. (2004). El salvaje metropolitano. Reconstrucción del conocimiento social en el trabajo de campo. Editorial Paidós. Buenos Aires.

Ibañez, N. (2002). Las Emociones en el Aula. Rev. Estudios Pedagógicos, n.28, 31-45. Valdivia.

Ibañez, N. (2001). El Contexto interaccional en el aula: una nueva dimensión evaluativa. Universidad Austral de Chile. Facultad de Filosofía y Humanidades, Rev. Estudios Pedagógicos, n.27, 43-53. Valdivia.

Larrosa, J. (2001). Lenguaje y educación. Revista Brasilera de Educación. 16: 68-80.

Maturana, H. (2001). Emociones y lenguaje en la educación y política. Dolmen Ediciones S. A. Santiago de Chile.

Maturana, H. (1999). Transformación en la convivencia. Edit. Dolmen. Santiago de Chile.

Maturana, H. y Varela, F. (2008). De máquinas y seres vivos. Autopoiesis: la 
Organización de lo Vivo. Editorial Universitaria. Santiago de Chile.

Miranda, Luis. (2011). Lenguaje: algo más que un mecanismo para la comunicación. Revista electrónica Educare. 15(1):161-170.

Morin, E. (1999). Los Siete saberes de la educación del futuro. Francia: Unesco.

Mulsow, G. (2000). Desarrollo humano: a través de los objetivos fundamentales transversales. Estudios Pedagógicos. Facultad de Filosofía y Humanidades. Universidad Austral de Chile. No 26.

Niebles, A. (2010). Cuerpos vividos y dinámicas relacionales en el escenario escolar: una mirada desde la fenomenología. Tesis para optar el grado de Magister en Desarrollo Humano. Valdivia: Facultad de Filosofía y Humanidades. Valdivia Chile.

Quintanilla, P. (2004). Comprender al otro es crear un espacio compartido: caridad, empatía y triangulación. Ideas y Valores. Departamento de Humanidades. Pontificia Universidad Católica del Perú, Revista Colombiana de Filosofía. 125: 81-97.

Pizarro, P. (2011). Creencias de los profesores respecto al desarrollo de competencias socioemocionales y su relación con el aprendizaje en estudiantes de $5^{\circ}$ y $6^{\circ}$ básico. En J.M. Román, M.A. Carbonero y J.D. Valdivieso (Comp.), Educación, aprendizaje y desarrollo en una sociedad multicultural (4105-4123). Madrid: Asociación Psicología y Educación y Colegio Oficial de Psicólogos de Castilla y León. ISBN978-84-614-82-96-2.
Saavedra, (2005). Conocimiento y desarrollo emocional desde el enfoque cognitivo, procesal sistémico. Universidad de Tarapacá, Límite. Revista de Filosofía y Psicología. Vol 1. N5: 31-45.

Santos, M. (2013). Manual de mapeo colectivo. Recursos cartográficos críticos para procesos territoriales de creación colaborativa. $1^{\circ}$ Edición. Buenos Aires.

Toro, S. (2005). Desarrollo humano y motricidad: una aproximación desde la empatía. En acción 1, Consentido. $1^{\circ}$ Edición. Universidad del Cauca. Toro, S. y Niebles, A. (2013). Corporeidad y aprendizaje en el contexto de la enseñanza general básica: comprensión y descripción de los procesos de construcción de conocimiento desde la acción relacional de los actores. Universidad Austral de Chile. Facultad de Filosofía y Humanidades, Rev. Estudios Pedagógicos, vol. XXXIX, n.1, 269284. Valdivia.

Varela, F. (2010). Fenómeno de la vida. JC Saéz. LOM Ediciones. Santiago. Chile.

Vasilachis, I. (2006). Estrategias de investigación cualitativa. Gedisa Ediciones. $1^{\circ}$ Edición. Barcelona.

Vigostky, L. (1988). El Desarrollo de los procesos psicológicos superiores. Editorial Crítica, Grupo editorial Grijalbo. México. 


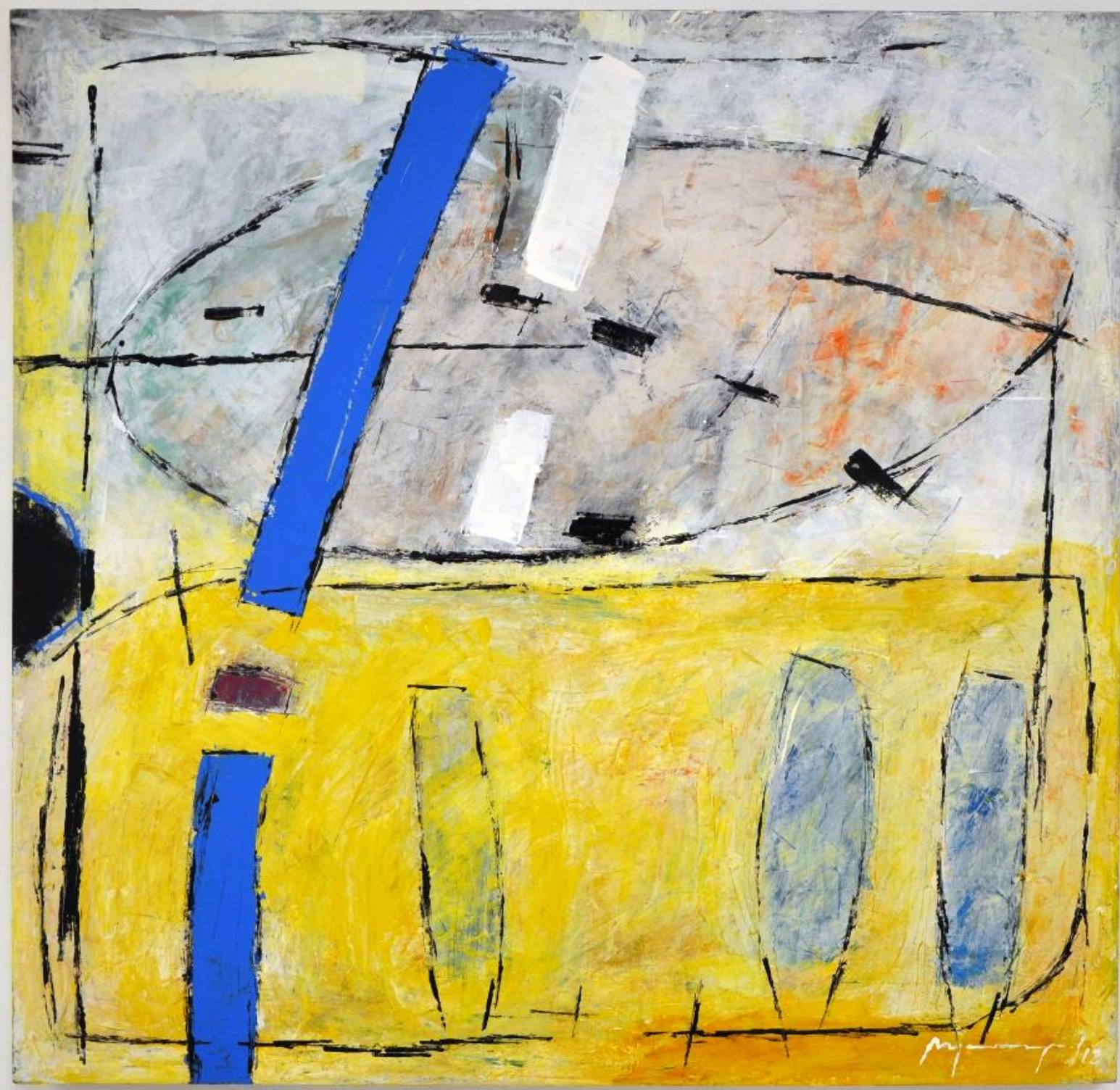

Título de la obra: Señales

Técnica: pigmentos industriales sobre polietileno

Dimensiones de la obra: $80 \mathrm{cms}$ x $80 \mathrm{cms}$

Añ $0: 2012$

Nombre de la imagen adjunta: 006 